\title{
On the Kontsevich integral of Brunnian links
}

\author{
KAZUO HABIRO \\ JEAN-BAPTISTE MEILHAN
}

\begin{abstract}
The purpose of the paper is twofold. First, we give a short proof using the Kontsevich integral for the fact that the restriction of an invariant of degree $2 n$ to $(n+1)-$ component Brunnian links can be expressed as a quadratic form on the Milnor $\bar{\mu}$ link-homotopy invariants of length $n+1$. Second, we describe the structure of the Brunnian part of the degree- $2 n$ graded quotient of the Goussarov-Vassiliev filtration for $(n+1)$-component links.
\end{abstract}

57M25, 57M27

\section{Introduction}

The notion of Goussarov-Vassiliev finite type link invariants provides a unifying viewpoint on the various quantum link invariants; see Bar-Natan [1], Goussarov [10; 11], Vassiliev [28]. The definition mainly relies on a descending filtration

$$
\mathbb{Z} \mathcal{L}(m)=J_{0}(m) \supset J_{1}(m) \supset \cdots
$$

of the free abelian group $\mathbb{Z} \mathcal{L}(m)$ generated by the set $\mathcal{L}(m)$ of isotopy classes of $m-$ component, oriented, ordered links in $S^{3}$. Here each $J_{n}(m)$ is generated by alternating sums of links over $n$ independent crossing changes. A homomorphism from $\mathbb{Z} \mathcal{L}(m)$ to an abelian group is said to be an invariant of degree $\leq n$ if it vanishes on $J_{n+1}(m)$. The Kontsevich integral [17] is a universal rational-valued finite type link invariant.

A link in $S^{3}$ is said to be Brunnian if every proper sublink of it is an unlink. The first author [14] proved that an $(n+1)$-component Brunnian link cannot be distinguished from the unlink by any invariant of degree $<2 n$ with values in any abelian group. In [16] the authors study the behavior of the invariants of degree $2 n$ on $(n+1)$-component Brunnian links.

The purpose of this paper is twofold. First, we give an alternative, shorter proof using the Kontsevich integral of Theorem 1.1, originally proved in [16], which relates the degree-2n invariants for $(n+1)$-component links to Milnor $\bar{\mu}$ link-homotopy 
invariants. Second, we describe the structure of the Brunnian part of the degree- $2 n$ graded quotient of the Goussarov-Vassiliev filtration for $(n+1)$-component links.

For any $(n+1)$-component Brunnian link $L$, the Milnor $\bar{\mu}$ link-homotopy invariants $\bar{\mu}_{i_{1}, \ldots, i_{n+1}}(L) \in \mathbb{Z}$ for nonrepeating indices $i_{1}, \ldots, i_{n+1} \in\{1,2, \ldots, n+1\}$ are welldefined and give a complete set of link-homotopy invariants [24]. It is also known that there are exactly $(n-1)$ ! independent invariants

$$
\bar{\mu}_{\sigma}(L):=\bar{\mu}_{\sigma(1), \sigma(2), \ldots, \sigma(n-1), n, n+1}(L) \in \mathbb{Z} \quad \text { for } \sigma \in S_{n-1},
$$

where $S_{n-1}$ denotes the symmetric group on $\{1,2, \ldots, n-1\}$.

Theorem 1.1 ([16, Theorem 1.2], stated slightly differently) Let $n \geq 2$ and let $f: \mathbb{Z} \mathcal{L}(n+1) \rightarrow \mathbb{Z}$ be any link invariant of degree $2 n$. Then there exist integers $f_{\sigma, \sigma^{\prime}}$ for $\sigma, \sigma^{\prime} \in S_{n-1}$ such that for any $(n+1)$-component Brunnian link $L$, we have

$$
f(L)-f(U)=\frac{1}{2} \sum_{\sigma, \sigma^{\prime} \in S_{n-1}} f_{\sigma, \sigma^{\prime}} \bar{\mu}_{\sigma}(L) \bar{\mu}_{\sigma^{\prime}}(L),
$$

where $U$ is the $(n+1)$-component unlink. Moreover, we have $f_{\sigma, \sigma^{\prime}}=f_{\sigma^{\prime}, \sigma}$ and $f_{\sigma, \sigma} \in 2 \mathbb{Z}$ for all $\sigma, \sigma^{\prime} \in S_{n-1}$.

Theorem 1.1 is proved in Section 2. The coefficients $f_{\sigma, \sigma^{\prime}}$ are defined there using the weight system of $f$. It follows from the arguments of Section 3 that we have $f_{\sigma, \sigma^{\prime}}=f_{\eta \sigma, \eta \sigma^{\prime}}$ for every $\eta \in S_{n-1}$. Thus there are integers $g_{\tau}$ for $\tau \in S_{n-1}$ satisfying $g_{\tau}=g_{\tau^{-1}}$ and $g_{1} \in 2 \mathbb{Z}$, such that $f_{\sigma, \sigma^{\prime}}=g_{\sigma^{-1} \sigma^{\prime}}$. Further, $f$ does not depend on the order of components for Brunnian links.

In Section 3 we give, in theory, a necessary and sufficient condition to extend a quadratic form of Milnor invariants to a link invariant of degree $\leq 2 n$. More precisely, we study the Brunnian part $\operatorname{Br}\left(\bar{J}_{2 n}(n+1)\right)$ of the graded quotient $\bar{J}_{2 n}(n+1)=$ $J_{2 n}(n+1) / J_{2 n+1}(n+1)$, which is defined as the subgroup generated by the elements $[L-U]_{J_{2 n+1}}$, where $L$ is Brunnian. We construct a homomorphism

$$
h_{n}: \mathcal{A}_{n-1}^{c}(\varnothing) \rightarrow \operatorname{Br}\left(\bar{J}_{2 n}(n+1)\right),
$$

where $\mathcal{A}_{n-1}^{c}(\varnothing)$ is a $\mathbb{Z}$-module of connected trivalent diagrams with $2 n-2$ vertices. We show that $h_{n}$ is surjective for $n \geq 3$ and is an isomorphism over $\mathbb{Q}$ for $n \geq 2$.

A few remarks are in order.

Remark 1.2 The original proof of Theorem 1.1 involved calculus of claspers. It provided, as a byproduct, a number of results on Brunnian (string) links and the linkhomotopy relation. The proof given here is shorter than the original one and may also look simpler depending on the reader's point of view. 
Remark 1.3 Recall that Milnor invariants of length $n+1$ for string links are Goussarov-Vassiliev invariants of degree $\leq n$; see Bar-Natan [2] and Lin [22]. For links, Milnor invariants are not well-defined in general. However, Theorem 1.1 shows that a quadratic expression in Milnor invariants, which is well-defined at least for $(n+1)$-component Brunnian links, may extend to a link invariant of degree $\leq 2 n$.

For example, the case $n=2$ of Theorem 1.1 states that any degree-4 invariant of 3 -component links restricts for Brunnian links to a scalar multiple of the square of the triple Milnor invariant $\mu_{123}(L)$. Recall that $\mu_{123}(L)^{2}$ for 3-component algebraically split links extends to the degree-4 coefficient of the Alexander-Conway polynomial, which is a degree-4 invariant; see Cochran [8].

Remark 1.4 Recall that trivalent diagrams appear in the study of Ohtsuki finite type invariants of integral homology spheres; see Ohtsuki [25], Garoufalidis and Ohtsuki [9] and Le [18]. The relation between Theorem 3.2 and Ohtsuki finite type invariants is discussed in [23].

Acknowledgments The authors wish to thank Thang Le and Christine Lescop for helpful comments and conversations. The first author is partially supported by the Japan Society for the Promotion of Science, Grant-in-Aid for Young Scientists (B), 16740033. The second author is supported by a Postdoctoral Fellowship and a Grant-in-Aid for Scientific Research of the Japan Society for the Promotion of Science.

\section{Proof of Theorem 1.1 using the Kontsevich integral}

In this section, we give a proof of Theorem 1.1 using the Kontsevich integral. For an introduction to the Kontsevich integral; see for example Bar-Natan [1], Chmutov and Duzhin, [7], Lescop [21] and Ohtsuki [26].

\subsection{Spaces of diagrams}

We recall the definition of the spaces of diagrams in which the Kontsevich integral takes its values.

A unitrivalent diagram (also called Feynman diagram or Jacobi diagram) is a finite graph $\Gamma$ with univalent and trivalent vertices such that each trivalent vertex is equipped with a cyclic order of the three incident edges. Throughout the paper, we assume that every component of a unitrivalent diagram has at least one univalent vertex. The degree of $\Gamma$ is half the number of vertices in $\Gamma$.

Algebraic $8 \mathcal{G}$ Geometric Topology, Volume 6 (2006) 
A unitrivalent diagram $\Gamma$ is on a 1 -manifold $X$ if the univalent vertices of $\Gamma$ are identified with distinct points on $X$ up to isotopy. Let $\mathcal{A}_{k}(X)$ denote the $\mathbb{Z}$-module generated by diagrams on $X$ of degree $k$, subject to the FI relations and the $S T U$ relations; see Figure 1.

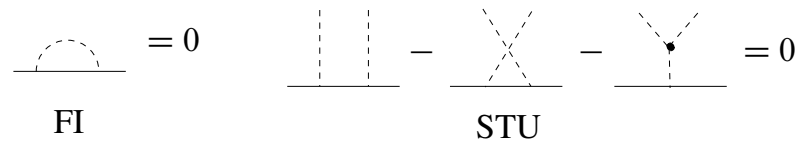

Figure 1

As is well-known, the $A S$ relations and the IHX relations depicted in Figure 2 are valid in $\mathcal{A}_{k}(X)$.

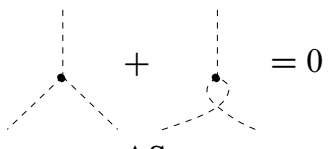

AS

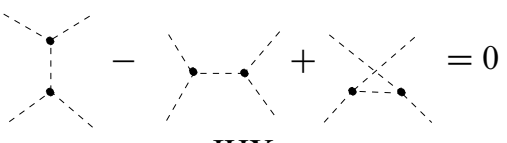

IHX

Figure 2

If $X$ is the disjoint union of $m$ copies of $S^{1}$, then $\mathcal{A}_{k}(X)$ is denoted also by $\mathcal{A}_{k}(m)$.

An open unitrivalent diagram for $m$ colors $\{1, \ldots, m\}$ is a unitrivalent graph such that each univalent vertex is labeled by an element of $\{1, \ldots, m\}$. Let $\mathcal{B}_{k}(m)$ denote the $\mathbb{Q}$-vector space generated by open unitrivalent diagrams of degree $k$ for $m$ colors $\{1, \ldots, m\}$, modulo the AS and the IHX relations.

Let $\sqcup^{m} I$ denote the disjoint union of $m$ copies of the unit interval $I$. There is a standard isomorphism $(m \geq 1)$

$$
\chi_{k}: \mathcal{B}_{k}(m) \stackrel{\simeq}{\longrightarrow} \mathcal{A}_{k}\left(\sqcup^{m} I\right) \otimes \mathbb{Q},
$$

called the Poincaré-Birkhoff-Witt isomorphism; see Bar-Natan [1].

For $m=1, \mathcal{A}_{k}(I)$ is isomorphic to $\mathcal{A}_{k}(1)$. For $m>1$, let $\mathcal{B}_{k}^{l}(m)$ denote the quotient of $\mathcal{B}_{k}(m)$ by all link relations (see Section 5.2 of Bar-Natan, Garoufalidis, Rozansky and Thurston [4]). The isomorphism $\chi_{k}$ induces a well-defined isomorphism

$$
\chi_{k}: \mathcal{B}_{k}^{l}(m) \stackrel{\simeq}{\longrightarrow} \mathcal{A}_{k}(m) \otimes \mathbb{Q} .
$$




\subsection{Proof of Theorem 1.1}

Suppose $L$ is an $(n+1)$-component Brunnian link in $S^{3}$. By [14, Proposition 12], there is an $(n+1)$-component Brunnian string link $T$ whose closure is $L$. (A string link is said to be Brunnian if every proper subtangle of it is a trivial string link.) Consider the Kontsevich integral $Z(T)$ of $T$, which lives in the completed $\mathbb{Q}$-vector space $\mathcal{A}\left(\sqcup^{n+1} I\right)=\prod_{k \geq 0} \mathcal{A}_{k}\left(\sqcup^{n+1} I\right) \otimes \mathbb{Q}$. Recall that $\mathcal{A}\left(\sqcup^{n+1} I\right)$ has an algebra structure with multiplication given by the 'stacking product' and with unit 1 given by the empty unitrivalent diagram.

Let $p: \mathcal{A}\left(\sqcup^{n+1} I\right) \simeq \mathcal{B}(n+1)$ denote the inverse of the Poincaré-Birkhoff-Witt isomorphism, where $\mathcal{B}(n+1)$ is the completed $\mathbb{Q}$-vector space $\prod_{k \geq 0} \mathcal{B}_{k}(n+1)$. Since $p(Z(T)) \in \mathcal{B}(n+1)$ is grouplike (see Le, Murakami and Ohtsuki [20]), we have

$$
p(Z(T))=\exp _{\sqcup}(P), \quad P \in \mathcal{P}(n+1),
$$

where $\mathcal{P}(n+1)$ denotes the primitive part of $\mathcal{B}(n+1)$, generated by connected diagrams and where $\exp _{\sqcup}$ denotes the exponential with respect to disjoint union of diagrams.

For each $i=1, \ldots, n+1$, consider the operation $\epsilon_{i}$ of omitting the $i$-th string. At the level of the string link we have $\epsilon_{i}(T)=\mathbf{1}_{n}$, since $T$ is Brunnian. Hence we have $\epsilon_{i}(P)=0$. It follows that $P$ can be expressed as an infinite $\mathbb{Q}$-linear combination of connected diagrams, each having at least one univalent vertex of color $i$. Since we have this property for $i=1, \ldots, n+1$, we can deduce that $P$ is an infinite $\mathbb{Q}$-linear combination of connected diagrams, each involving all the colors.

By an easy counting argument, we see that $P$ can be expressed as

$$
P=P_{n}+P_{n+1}+P_{n+2}+\cdots
$$

where $P_{n}$ is a linear combination of trees of degree $n$, and $P_{k}$ for $k>n$ is a linear combination of connected diagrams of degree $k$. Moreover, we can use the AS and IHX relation to express $P_{n}$ as a linear combination of trees $t_{\sigma}$ as depicted in Figure 3, $\sigma \in S_{n-1}$. By Habegger and Masbaum's result [13], $P_{n}$ is thus a $\mathbb{Z}$-linear combination

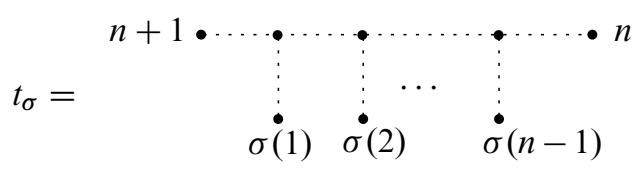

Figure 3

of trees which corresponds to the Milnor invariants of length $n+1$ of the form 
$\bar{\mu}_{\sigma}:=\bar{\mu}_{\sigma(1), \sigma(2), \ldots, \sigma(n-1), n, n+1}$, for $\sigma \in S_{n-1}$. More precisely, from [13, Theorem 6.1 and Proposition 7.1] and properties of the Milnor $\bar{\mu}$ link-homotopy invariants [24] it follows that

$$
P_{n}=\sum_{\sigma \in S_{n-1}} \bar{\mu}_{\sigma}(L) t_{\sigma}
$$

By (2-1), we have

$$
\begin{aligned}
p(Z(T)) & =\exp _{\sqcup}\left(P_{n}+P_{n+1}+\cdots+P_{2 n}+\cdots\right) \\
& =1+P_{n}+P_{n+1}+\cdots+P_{2 n-1}+\left(P_{2 n}+\frac{1}{2} P_{n}^{2}\right)+O_{>2 n},
\end{aligned}
$$

where $O_{>2 n}$ denotes a sum of terms of degree greater than $2 n$. Hence we have

$$
Z(T)=1+P_{n}^{\prime}+P_{n+1}^{\prime}+\cdots+P_{2 n-1}^{\prime}+P_{2 n}^{\prime}+p^{-1}\left(\frac{1}{2} P_{n}^{2}\right)+O_{>2 n},
$$

where $P_{k}^{\prime}=p^{-1}\left(P_{k}\right)$ for $k=n, \ldots, 2 n$.

By [19, Theorem 6.1], the Kontsevich integral $Z(L)$ of $L$ can be obtained from $Z(T)$ by first multiplying by the $(n+1)$-parallel $\Delta^{n}(Z(\circlearrowleft))$ of the Kontsevich integral of the unknot $Z(\circlearrowleft) \in \mathcal{A}(I)$ and then mapping into the space $\mathcal{A}(n+1)$ by closing the strings. Thus we have

$$
Z(L)-Z(U)=\pi\left(\Delta^{n}(Z(\circlearrowleft))(Z(T)-1)\right),
$$

where $U$ denotes the $(n+1)$-component unlink in the 3-sphere $S^{3}$ and where the projection $\pi: \mathcal{A}\left(\sqcup^{n+1} I\right) \rightarrow \mathcal{A}(n+1)$ is induced by the closure operation.

As is well-known $[3 ; 27 ; 5]$,

$$
Z(\circlearrowleft)=1+W_{2}+W_{4}+W_{6}+\cdots
$$

where $W_{2 j}$ is a linear combination of unitrivalent diagram of degree $2 j$ consisting of finitely many wheels, ie, connected unitrivalent diagrams of Euler number 1.

By (2-3), (2-4) and (2-5), we have

$$
Z(L)-Z(U)=\frac{1}{2} \pi p^{-1}\left(P_{n}^{2}\right)+\sum_{i=n}^{2 n} \pi\left(P_{i}^{\prime}\right)+\sum_{i, j} \pi\left(P_{i}^{\prime} \Delta^{n}\left(W_{2 j}\right)\right)+O_{>2 n},
$$

where the second sum is over $i, j$ with $n \leq i \leq 2 n, 2 \leq 2 j \leq n, i+2 j \leq 2 n$.

One can verify that the two sums in the right-hand side of (2-6) vanish, using the fact that $\pi$ kills each diagram with at least one string having exactly one univalent vertex. Thus we have

$$
Z(L)-Z(U)=\frac{1}{2} \pi p^{-1}\left(P_{n}^{2}\right)+O_{>2 n}
$$


It follows that the first nontrivial, $\mathbb{Z}$-valued, finite type invariants of $(n+1)$-component Brunnian links are of degree $2 n$. Moreover, (2-2) and (2-7) imply that

$$
Z(L)-Z(U)=\frac{1}{2} \sum_{\sigma, \sigma^{\prime} \in S_{n-1}} \bar{\mu}_{\sigma}(L) \bar{\mu}_{\sigma^{\prime}}(L) \pi p^{-1}\left(t_{\sigma} t_{\sigma^{\prime}}\right)+O_{>2 n} .
$$

Let $f$ be a $\mathbb{Z}$-valued link invariant of degree $2 n$, and denote by $W_{f}$ the weight system of $f$. To complete the proof of Theorem 1.1, we obtain (1-2) by setting

$$
f_{\sigma, \sigma^{\prime}}=W_{f}\left(\pi p^{-1}\left(t_{\sigma} t_{\sigma^{\prime}}\right)\right) .
$$

Remark 2.1 Obviously, we have $f_{\sigma, \sigma^{\prime}}=f_{\sigma^{\prime}, \sigma}$ for all $\sigma, \sigma^{\prime} \in S_{n-1}$. Observe that the identity depicted in Figure 4 can be derived from the STU relation. This identity implies that, for any $\sigma \in S_{n-1}$, we have $\pi p^{-1}\left(t_{\sigma}^{2}\right)=2 d_{\sigma}$ for some $d_{\sigma} \in \mathcal{A}_{2 n}(n+1)$. Hence $f_{\sigma, \sigma}=W_{f}\left(2 d_{\sigma}\right)$ is even.

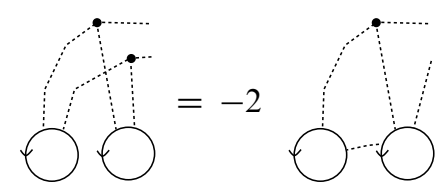

Figure 4: Hint: use the fact that, thanks to the STU relation, a circle component inserted in an edge attached to a trivalent vertex $v$ can be moved to another edge attached to $v$ (see Figure 5).

Remark 2.2 As recalled in the introduction, it is known that no Goussarov-Vassiliev invariant of degree $<2 n$ with values in any abelian group can distinguish $L$ from $U$ [14]. The above proof of Theorem 1.1 recovers, using the Kontsevich integral, a weaker version (namely, a rational version) of this result.

\section{The Brunnian part of $\bar{J}_{2 n}(n+1)$}

The purpose of this section is to give an almost complete description of the structure of the Brunnian part $\operatorname{Br}\left(\bar{J}_{2 n}(n+1)\right)$ of the $2 n$-th graded quotient $\bar{J}_{2 n}(n+1)$ of the Goussarov-Vassiliev filtration for $(n+1)$-component links, using the $\mathbb{Z}$-module $\mathcal{A}_{n-1}^{c}(\varnothing)$ of connected trivalent diagrams.

Algebraic $8 \mathcal{G}$ Geometric Topology, Volume 6 (2006) 


\subsection{Claspers and Goussarov-Vassiliev filtration}

The Goussarov-Vassiliev filtration (1-1) is usually defined in terms of singular links, where $J_{k}(m) \subset \mathbb{Z} \mathcal{L}(m)$ is generated by the $k$-fold alternating sums of links determined by singular links with $k$ double points; see eg Bar-Natan [1]. One can also redefine the filtration using claspers; see Habiro [15] and Goussarov [12]. Here we will freely use the definitions and conventions of [15]. Recall that a graph scheme of degree $k$ for a link $L$ in $S^{3}$ is a set $S=\left\{G_{1}, \ldots, G_{l}\right\}$ of $l$ disjoint (strict) graph claspers $G_{1}, \ldots, G_{l}$ for $L$ such that $\sum_{i=1}^{l} \operatorname{deg} G_{i}=k$. We set

$$
[L, S]=\left[L ; G_{1}, \ldots, G_{l}\right]:=\sum_{S^{\prime} \subset S}(-1)^{|S|-\left|S^{\prime}\right|} L_{\cup S^{\prime}} \in \mathbb{Z} \mathcal{L}(m),
$$

where the sum runs over all subsets $S^{\prime}$ of $S,\left|S^{\prime}\right|$ denote the number of elements of $S^{\prime}$, and $L \cup S^{\prime}$ denotes the result from $L$ of surgery along the union of elements in $S^{\prime}$. It is known that $J_{k}(m)$ is generated by the elements of the form $[L, S]$, where $L \in \mathcal{L}(m)$ and $S$ is a graph scheme for $L$ of degree $k$ [15].

\subsection{The Brunnian part of the Goussarov-Vassiliev filtration}

If $L$ is an $(n+1)$-component Brunnian link in $S^{3}$, then, by [14, Theorem 3], we have $L-U \in J_{2 n}(n+1)$. Define $\operatorname{Br}\left(\bar{J}_{2 n}(n+1)\right)$ to be the $\mathbb{Z}$-submodule of $\bar{J}_{2 n}(n+1)$ generated by the elements $[L-U]_{J_{2 n+1}}$, where $L$ is an $(n+1)$-component Brunnian link. We call $\operatorname{Br}\left(\bar{J}_{2 n}(n+1)\right)$ the Brunnian part of $\bar{J}_{2 n}(n+1)$.

\subsection{Trivalent diagrams}

A trivalent diagram is a finite trivalent graph $\Gamma$ such that each vertex is equipped with a cyclic order on the three incident edges. The degree of $\Gamma$ is half the number of its vertices.

For $k \geq 0$, let $\mathcal{A}_{k}(\varnothing)$ denote the $\mathbb{Z}$-module generated by trivalent diagrams of degree $k$, subject to the AS and IHX relations (see Figure 2).

Let $\mathcal{A}_{n}^{c}(\varnothing)$ denote the $\mathbb{Z}$-submodule of $\mathcal{A}_{n}(\varnothing)$ generated by connected trivalent diagrams.

\subsection{The circle-insertion map $g_{n}$}

For $n \geq 2$, denote the homomorphism which maps each trivalent diagram $\Gamma$ to the result of inserting $n+1$ ordered copies of $S^{1}$ in its edges by

$$
g_{n}: \mathcal{A}_{n-1}^{c}(\varnothing) \rightarrow \mathcal{A}_{2 n}(n+1) .
$$




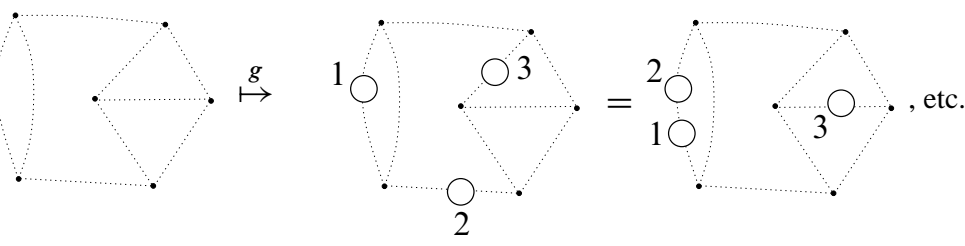

Figure 5

See Figure 5. This map is well-defined thanks to the STU relation, since $\Gamma$ is connected.

We need the following result.

Proposition 3.1 For $n \geq 2$, the map

$$
g_{n} \otimes \mathbb{Q}: \mathcal{A}_{n-1}^{c}(\varnothing) \otimes \mathbb{Q} \rightarrow \mathcal{A}_{2 n}(n+1) \otimes \mathbb{Q}
$$

is injective.

Proof Define a homomorphism

$$
P: \mathcal{B}_{2 n}^{l}(n+1) \longrightarrow \mathcal{A}_{n-1}(\varnothing) \otimes \mathbb{Q}
$$

as follows. Let $\Gamma$ be a diagram in $\mathcal{B}_{2 n}^{l}(n+1)$. If there are exactly two univalent vertices colored by $i$ for each $i=1, \ldots, n+1$, then we set $P(\Gamma)$ to be the trivalent diagram obtained from $\Gamma$ by joining each pair of univalent vertices of the same color. Otherwise, set $P(\Gamma)=0$. Let

$$
\pi: \mathcal{A}_{n-1}(\varnothing) \otimes \mathbb{Q} \longrightarrow \mathcal{A}_{n-1}^{c}(\varnothing) \otimes \mathbb{Q}
$$

denote the projection which maps each connected diagram into itself and maps nonconnected diagrams to 0 . One can easily check that the composition

$\mathcal{A}_{n-1}^{c}(\varnothing) \otimes \mathbb{Q} \stackrel{g_{n} \otimes \mathbb{Q}}{\longrightarrow} \mathcal{A}_{2 n}(n+1) \otimes \mathbb{Q} \stackrel{\chi_{2 n}^{-1}}{\longrightarrow} \mathcal{B}_{2 n}^{l}(n+1) \stackrel{P}{\longrightarrow} \mathcal{A}_{n-1}(\varnothing) \otimes \mathbb{Q} \stackrel{\pi}{\longrightarrow} \mathcal{A}_{n-1}^{c}(\varnothing) \otimes \mathbb{Q}$ is the identity. Hence $g_{n} \otimes \mathbb{Q}$ is injective.

\subsection{Structure of $\operatorname{Br}\left(\bar{J}_{2 n}(n+1)\right)$}

We need the well-known surjective surgery map

$$
\xi_{k}: \mathcal{A}_{k}(m) \rightarrow \bar{J}_{k}(m)
$$

which maps each unitrivalent diagram $\Gamma=\Gamma_{1} \cup \cdots \cup \Gamma_{l}$ to the coset of alternating sum $\left[U ; G_{1}, \ldots, G_{l}\right]$, where $G_{1}, \ldots, G_{l}$ are "realizations" of $\Gamma_{1}, \ldots, \Gamma_{l}$; see Figure 6 . 


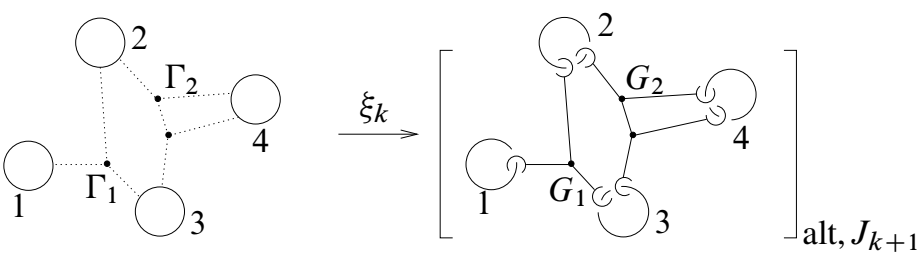

Figure 6: Realization of unitrivalent diagrams and the map $\xi_{k}$. Here $[-]_{\text {alt }, J_{k+1}}$ denotes the $J_{k+1}$-coset of the alternating sum of the form (3-1) defined by the link and graph claspers.

This map coincides with the standard map which "replaces chords with double points" [15, Section 8.2]. $\xi_{k} \otimes \mathbb{Q}$ is an isomorphism, with inverse given by the Kontsevich integral.

For $n \geq 2$, let $h_{n}$ denote the composition

$$
h_{n}: \mathcal{A}_{n-1}^{c}(\varnothing) \stackrel{g_{n}}{\longrightarrow} \mathcal{A}_{2 n}(n+1) \stackrel{\xi_{2 n}}{\longrightarrow} \bar{J}_{2 n}(n+1) .
$$

Theorem 3.2 (1) For $n \geq 3$ we have $h_{n}\left(\mathcal{A}_{n-1}^{c}(\varnothing)\right)=\operatorname{Br}\left(\bar{J}_{2 n}(n+1)\right)$. For $n=2$, $h_{2}\left(\mathcal{A}_{1}^{c}(\varnothing)\right)$ is an index 2 subgroup of $\operatorname{Br}\left(\bar{J}_{4}(3)\right)$.

(2) For $n \geq 2$, the $\mathbb{Q}$-linear map

$$
h_{n} \otimes \mathbb{Q}: \mathcal{A}_{n-1}^{c}(\varnothing) \otimes \mathbb{Q} \rightarrow \operatorname{Br}\left(\bar{J}_{2 n}(n+1)\right) \otimes \mathbb{Q}
$$

is an isomorphism.

Proof (1) Suppose $n \geq 3$. First we show that

$$
\operatorname{Br}\left(\bar{J}_{2 n}(n+1)\right) \subset h_{n}\left(\mathcal{A}_{n-1}^{c}(\varnothing)\right) .
$$

As outlined in [16, Section 7], $\operatorname{Br}\left(\bar{J}_{2 n}(n+1)\right)$ is $\mathbb{Z}$-spanned by

$$
\begin{aligned}
\frac{1}{2}\left[U ; T_{\sigma}, \widetilde{T}_{\sigma}\right] & \text { for } \sigma \in S_{n-1}, \\
{\left[U ; T_{\sigma}, \widetilde{T}_{\sigma^{\prime}}\right] } & \text { for } \sigma, \sigma^{\prime} \in S_{n-1},
\end{aligned}
$$

where for all $\sigma, \sigma^{\prime}$ in the symmetric group $S_{n-1}, T_{\sigma}$ is the simple degree- $n$ tree clasper for the $(n+1)$-component unlink $U$ depicted in Figure 7 , and $\widetilde{T}_{\sigma^{\prime}}$ is obtained from $T_{\sigma^{\prime}}$ by a small isotopy so that it is disjoint from $T_{\sigma} .\left(\frac{1}{2}\left[U ; T_{\sigma}, \widetilde{T}_{\sigma}\right]\right.$ means an element $x \in \operatorname{Br}\left(\bar{J}_{2 n}(n+1)\right)$ such that $2 x=\left[U ; T_{\sigma}, \widetilde{T}_{\sigma}\right]$. Existence of such an element is shown in [16].) 


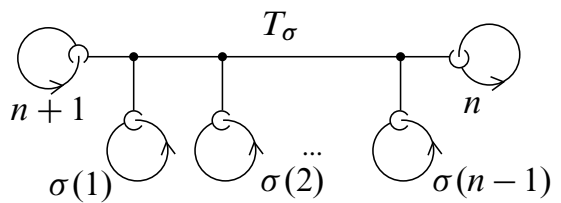

Figure 7: The tree clasper $T_{\sigma}$

For $n \geq 3$, one can check using the AS and IHX relations that $\frac{1}{2}\left[U ; T_{\sigma}, \widetilde{T}_{\sigma}\right]_{J_{2 n+1}}$ is equal to:

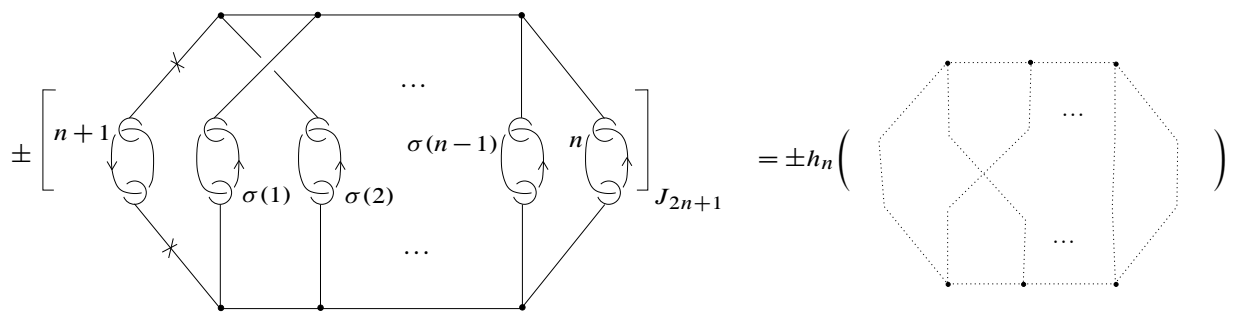

For $n \geq 2,\left[U ; T_{\sigma}, \widetilde{T}_{\sigma^{\prime}}\right]_{J_{2 n+1}}$ is equal to:

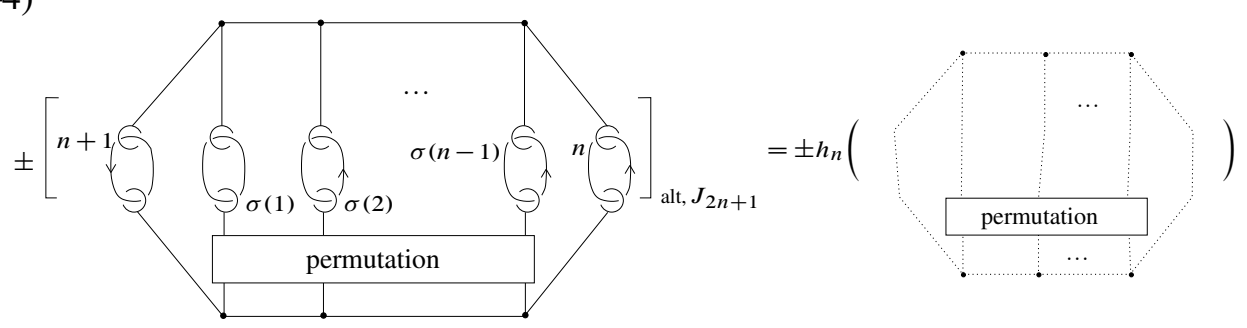

Hence we have (3-3) for $n \geq 3$.

Now we prove

$$
h_{n}\left(\mathcal{A}_{n-1}^{c}(\varnothing)\right) \subset \operatorname{Br}\left(\bar{J}_{2 n}(n+1)\right) .
$$

It suffices to prove that every element of $\mathcal{A}_{n-1}^{c}(\varnothing)$ is a $\mathbb{Z}$-linear combination of elements of the form depicted in the right hand side of (3-4). This follows from the fact that any chord diagram on a circle is, up to $4 T$ relations [1], a linear combination of chord diagrams of this form (where the dotted circle is replaced by a solid one). Chmutov and Duzhin proved a quite similar statement [6, Theorem 2.2], where they also assume the FI relation (see Figure 1). For trivalent diagrams, a $4 T$ relation can be easily derived from the IHX relation, but we do not have the FI relation. It is easy to avoid the latter in the proof of Chmutov-Duzhin (it is only used in one place [6, Section 3.4.2.2]) to prove the above-mentioned result on chord diagrams. 
The case $n=2$ follows, since $\operatorname{Br}\left(\bar{J}_{4}(3)\right)$ is $\mathbb{Z}$-spanned by $\left[U ; T_{1}\right]_{J_{5}}=\frac{1}{2}\left[U ; T_{1}, \widetilde{T}_{1}\right]_{J_{5}}$, and $h_{2}\left(\mathcal{A}_{1}^{c}(\varnothing)\right)$ is $\mathbb{Z}$-spanned by $\left[U ; T_{1}, \widetilde{T}_{1}\right]_{5}$. (Here ' 1 ' in ' $T_{1}$ ' denotes the unit in the (trivial) symmetric group $S_{1}$ of order 1 .)

(2) Since $\xi_{k}$ is an isomorphism over $\mathbb{Q}$, the assertion follows immediately from (1) above and Proposition 3.1.

Corollary 3.3 Let $f$ be a $\mathbb{Z}$-valued invariant of degree $2 n$ for $(n+1)$-component links in $S^{3}$. Then $f(L)$ restricted to Brunnian links is invariant under permutation of components and under orientation reversal of any component.

\section{References}

[1] D Bar-Natan, On the Vassiliev knot invariants, Topology 34 (1995) 423-472 MR1318886

[2] D Bar-Natan, Vassiliev homotopy string link invariants, J. Knot Theory Ramifications 4 (1995) 13-32 MR1321289

[3] D Bar-Natan, S Garoufalidis, L Rozansky, D P Thurston, Wheels, wheeling, and the Kontsevich integral of the unknot, Israel J. Math. 119 (2000) 217-237 MR1802655

[4] D Bar-Natan, S Garoufalidis, L Rozansky, D P Thurston, The Århus integral of rational homology 3-spheres. II. Invariance and universality, Selecta Math. (N.S.) 8 (2002) 341-371 MR1931168

[5] D Bar-Natan, T T Q Le, D P Thurston, Two applications of elementary knot theory to Lie algebras and Vassiliev invariants, Geom. Topol. 7 (2003) 1-31 MR1988280

[6] S V Chmutov, S V Duzhin, An upper bound for the number of Vassiliev knot invariants, J. Knot Theory Ramifications 3 (1994) 141-151 MR1279917

[7] S Chmutov, S Duzhin, The Kontsevich integral, Acta Appl. Math. 66 (2001) 155-190 MR1837618

[8] T D Cochran, Concordance invariance of coefficients of Conway's link polynomial, Invent. Math. 82 (1985) 527-541 MR811549

[9] S Garoufalidis, T Ohtsuki, On finite type 3-manifold invariants. III. Manifold weight systems, Topology 37 (1998) 227-243 MR1489202

[10] M N Goussarov, A new form of the Conway-Jones polynomial of oriented links, Zap. Nauchn. Sem. Leningrad. Otdel. Mat. Inst. Steklov. (LOMI) 193 (1991) 4-9, 161 MR1157140

[11] M Goussarov, On n-equivalence of knots and invariants of finite degree, from: "Topology of manifolds and varieties", Adv. Soviet Math. 18, Amer. Math. Soc., Providence, RI (1994) 173-192 MR1296895 
[12] M N Goussarov, Variations of knotted graphs. The geometric technique of n-equivalence, Algebra i Analiz 12 (2000) 79-125 MR1793618

[13] N Habegger, G Masbaum, The Kontsevich integral and Milnor's invariants, Topology 39 (2000) 1253-1289 MR1783857

[14] K Habiro, Brunnian links, claspers, and Goussarov-Vassiliev finite type invariants, to appear in Math. Proc. Camb. Phil. Soc.

[15] K Habiro, Claspers and finite type invariants of links, Geom. Topol. 4 (2000) 1-83 MR1735632

[16] K Habiro, J-B Meilhan, Finite type invariants and Milnor invariants for Brunnian links arXiv:math.GT/0510534

[17] M Kontsevich, Vassiliev's knot invariants, from: “I. M. Gel'fand Seminar", Adv. Soviet Math. 16, Amer. Math. Soc., Providence, RI (1993) 137-150 MR1237836

[18] T T Q Le, An invariant of integral homology 3-spheres which is universal for all finite type invariants, from: "Solitons, geometry, and topology: on the crossroad", Amer. Math. Soc. Transl. Ser. 2 179, Amer. Math. Soc., Providence, RI (1997) 75-100 MR1437158

[19] T T Q Le, J Murakami, Parallel version of the universal Vassiliev-Kontsevich invariant, J. Pure Appl. Algebra 121 (1997) 271-291 MR1477611

[20] T T Q Le, J Murakami, T Ohtsuki, On a universal perturbative invariant of 3 manifolds, Topology 37 (1998) 539-574 MR1604883

[21] C Lescop, Introduction to the Kontsevich integral of framed tangles, lecture notes (1999)

[22] X-S Lin, Power series expansions and invariants of links, from: "Geometric topology (Athens, GA, 1993)”, AMS/IP Stud. Adv. Math. 2, Amer. Math. Soc., Providence, RI (1997) 184-202 MR1470727

[23] J-B Meilhan, On surgery along Brunnian links in 3-manifolds arXiv: math.GT/0603421

[24] J Milnor, Link groups, Ann. of Math. (2) 59 (1954) 177-195 MR0071020

[25] T Ohtsuki, Finite type invariants of integral homology 3-spheres, J. Knot Theory Ramifications 5 (1996) 101-115 MR1373813

[26] T Ohtsuki, Quantum invariants, Series on Knots and Everything 29, World Scientific Publishing Co., River Edge, NJ (2002) MR1881401

[27] D P Thurston, Wheeling: A diagrammatic analogue of the Duflo isomorphism, $\mathrm{PhD}$ thesis, University of California Berkeley (2000) arXiv:math.QA/0006083

[28] V A Vassiliev, Cohomology of knot spaces, from: "Theory of singularities and its applications”, Adv. Soviet Math. 1, Amer. Math. Soc., Providence, RI (1990) 23-69 MR1089670 
Research Institute for Mathematical Sciences, Kyoto University

Kyoto 606-8502, Japan

habiro@kurims.kyoto-u.ac.jp, meilhan@kurims.kyoto-u.ac.jp

Received: 10 January 2006 Revised: 3 July 2006

Algebraic 83 Geometric Topology, Volume 6 (2006) 